\title{
Human resource performance predictors based on the human energy profile
}

\author{
Andronicus TORP \\ Politehnica University of Bucharest, Bucharest, Romania \\ andronicus.torp@icloud.com \\ Andreia Gabriela ANDREI \\ Alexandru Ioan Cuza University of Iasi, Iasi, Romania \\ andrei.andreia@gmail.com \\ Anca Alexandra PURCAREA \\ Politehnica University of Bucharest, Bucharest, Romania \\ apurcarea@gmail.com
}

\begin{abstract}
This paper is a comparative study on the findings regarding the connection between a person's energy profile and that person's professional performance. As the performance predictors that are used within Human Resource Management may provide a company with important information regarding the future performance of an employee, it is of great importance that these performance predictors be kept up-to-date, both in what regards the precision of each predictor, and by including new performance predictors to the present array of HR predictors should such new predictors be found. This paper is an empirical examination of two such predictors, stress and energy, and argues that, based on the available empirical material, it seems to be possible to expand the present selection of HR predictors with these two predictors as well. This study is based on the ontological framework set forth by academics such as Einstein, Hawking, Tiller, Hunt, Motoyama, regarding the possibility of assessing the human being based on their energy profile. The part concerning Human Resource Management is based on the scientific framework put forth by Hunter \& Hunter. Their study shows the validity of the vast majority of the performance predictors used within Human Resource Management, and discusses their practical validity. Then, there is the trans-disciplinary approach, where it is shown based on the empirical studies conducted by Torp et al. if, and how, the present array of performance indicators that are used in the field of Human Resource Management may be improved. Here, different and complementary scientific studies are included to document that the proposed Human Resource Management performance predictor is in reality more than just a predictor, it is an assessment tool that can both predict, and at the same time help quantify a series of the most modern initiatives within Human Resource Management, such as integrating sport, mindfulness, diet, etc. in the workday in order to improve performance.
\end{abstract}

Keywords: Assessment, Electrophotonic Imaging, Energy, Human Resource Management, Performance, Stress.

\section{Introduction}

According to Boxall and Purcell, HR is one out of four fundamental pillars of any company; the others being marketing, production, and finance (Boxall \& Purcell, 2011). They argue that if you remove one of these pillars, any company will collapse or, at least, become a holding company without any activity. Thus, the human resources, not just the people, but the right people with the KSAOs that they possess, manifested in a manner that helps the company to obtain its strategic goals, are of vital importance to the company. To ensure the 
on-boarding of these "right people", certain performance predictors have been developed over the last 100 years, which is generally considered to be the lifetime of Human Resource Management as an academic field (Lengnick-Hall et al., 2009).

These performance predictors are, amongst others, Cognitive Ability, Experience, Job try out, Education, Grades, Age, etc. It probably goes without saying that some of these predictors are better than others, however, according to Vinchur \& Bryan (in Schmitt, 2012) the list of performance predictors is pretty much the same today as it was 100 years ago.

It is, as Niels Bohr might have said, interesting, that in a world that is so often referred to as changing and undergoing rapid transformation the methods that are used to choose the highest performers in a company are not undergoing the same rapid transformation, at least in order to keep up with the development in the world.

This article aims at comparing the present array of performance predictors with two completely new performance predictors, that of energy and stress, assessed with the Electrophotonic Imaging (EPI) Device, in order to understand their place amongst the present array of performance predictors. These two performance predictors, stress and energy, assessed with the EPI Device are, amongst others (Bundzen et al., 2004; Drozdovski et al., 2012), used with great success in the field of sports, and although differences will probably be found between what predicts superior performance in sports versus in a professional context, it seems worthwhile examining if, and in case so, how, these predictors may find use within the field of Human Resource Management.

\section{Literature review}

Probably the largest and most famous scientific meta-study ever conducted regarding the validity of performance predictors is that of Hunter \& Hunter (1984). What they came up with was the following list of performance predictors that is shown in table 1 :

Table 1. Performance predictors according to Hunter \& Hunter

\begin{tabular}{|l|l|}
\hline Performance predictors & Mean validity (Hunter \& Hunter, 1984) \\
\hline Ability composite & .53 \\
\hline Job try-out & .44 \\
\hline Biographical inventory & .37 \\
\hline Reference check & .26 \\
\hline Experience & .18 \\
\hline Interview & .14 \\
\hline Training and experience ratings & .13 \\
\hline Academic achievement (grades) & .11 \\
\hline Education (length) & .10 \\
\hline Interest & .10 \\
\hline Age & -.01 \\
\hline & \multicolumn{2}{|c|}{ Source: Hunter \& Hunter (1984) }
\end{tabular}

As it can be seen, the best performance predictor according to them is Cognitive ability (0.53), followed by Job try-out (.44), and on the third place Biographical inventory (.37). 
Interestingly enough, it seems that some of the performance predictors generally used in the recruitment process, like education and grades, are relatively weak in their prediction of future professional performance. Age is the worst "predictor", with -0.01, and thus it does not even deserve to be considered a predictor of performance.

Thus, it may be considered relevant to re-evaluate not just the present array of performance predictors, but the entire selection process, in order to base it on scientific findings, and not just on behaviour that is more or less unconsciously repeated over and over in selection processes.

\section{Methodology}

This work is based on a vast body of knowledge indicating that everything in this universe, including the human being, consists of energy (Bodanis, 2000; Hawking, 2010) and that this energy can be measured (Motoyama, 1978; Hunt, 1995; Korotkov, 2002, 2004). And not just measured, but scientists as Hunt and Tiller state that these measurements can be used to make predictions regarding, for example, the functioning of a human being, and also regarding behavioural traits.

According to Einstein $\mathrm{e}=\mathrm{mc}^{2}$ (in Bodanis, 2000), in other words, everything consists of energy. This "everything" must per definition also include the chair that you are sitting on, the paper that you are reading, your body, as well as your feelings, and even your thoughts. According to physics, there are four forces that rule this energy: gravity, the strong and the weak nuclear force, and finally electromagnetism. According to Hawking (2010), electromagnetism is the foundation of chemistry and biology, and since there is a general agreement that we human beings are biological entities with a chemical level, it should be possible to make objective and quantitative assessments regarding the human being founded at a deeper level than what such assessments normally yield.

There is already a list of scientists who have made such assessments, predominantly within medicine, and more recently yet in a somewhat smaller scale, sports. However, as the principle behind it is the same, such measurements should also be possible within professional performance, and thus Human Resource Management.

Probably the most famous scientist to work with this kind of energy assessment is Prof. Hunt from UCLA. Using equipment developed for NASA, she made a long list of studies regarding how the energy profile of a person connects with that person's health, yet she also made observations about the connection between the energy profile of a person and that person's behavioural tendencies, profession, interaction with other people, etc.

At least three of Prof. Hunt's findings may be considered relevant for predicting future performance, and thus they can be the foundation for an HR assessment method based on the human energy profile.

The first is that it is possible to predict the profession of a person based on that person's energy profile. Obviously, this does not necessarily mean that a person who has the energy profile of a specific profession is also a high performer in that profession, however, it is be likely that a person with the energy profile of a specific profession would be better in performing the activities connected with that profession than a person with the energy profile of another profession. Of course, further scientific studies are required in order to clarify this.

The second of Hunt's findings indicating a possible connection between a person's energy profile and professional performance is the fact that she writes that under UCLA 
they have a room that is not just shielded from external electromagnetic influences, but it is even possible to increase or decrease the electromagnetic field in that room. What she found was that when they increased the field in the room people became more coherent in their thoughts, lucid, and stronger. However, on the other hand, when they diminished the field, people became less coherent, mentally unclear, and weaker. Everything else being equal, it seems reasonable to assume that a person who is coherent in their thinking, lucid, and physically strong is more performant than a person who is incoherent, mentally unclear, and physically weak. Once more, further scientific studies are required to clarify this assumption.

The third, and final, of Hunt's findings that will be included here, because of its possible application within $\mathrm{HR}$, is that of the experiments that she did with putting people together while measuring their energy profile. She discovered that when doing so one out of three results would follow. Either the energy fields would completely refuse each other, or they would merge, yet at a higher level of vibration than each field individually or, the third option, one field would overtake and completely dominate the other. Unfortunately, it seems that her study did not include considerations regarding the nature of the interaction between test subjects, and it is possible that there is no connection between people's energy profile and their behaviour with respect to one another. However, it may be considered a possibility that this kind of study can be used to predict how different people interact together, and thus find use in HR, for example in order to put high performing teams together. It is a possibility that people whose energy fields completely refuse each other might not work very well together, and that there might be a certain asymmetric relationship between people in the cases where one energy field overtakes and dominates the other. Whereas it could be considered a possibility that the people whose fields would merge, yet at a much higher level of vibration than individually, would perform very well together. Once again, these are possibilities that need to be clarified through further scientific studies.

Based on this, it seems likely that it should be possible to make an HR assessment tool based on the human energy profile, and that such an assessment tool could expand the present array of HR performance predictors, and thus add something new to the field.

\section{Results and discussions}

Using the EPI Device Torp et al. (2017a, b) have shown that it is possible to make predictions regarding both individual professional performance and company performance, based on assessing the human energy profile.

In this study, Energy and Stress were assessed and the following hypotheses were made: Hypothesis 1: Energy influences Turnover.

Hypothesis 2: Energy influences Profit.

Hypothesis 3: Energy influences the Number of Transports.

Hypothesis 4: Stress influences Turnover.

Hypothesis 5: Stress influences Profit.

Hypothesis 6: Stress influences the Number of Transports.

This led to a conceptual model that looks like what is represented in figure 1: 


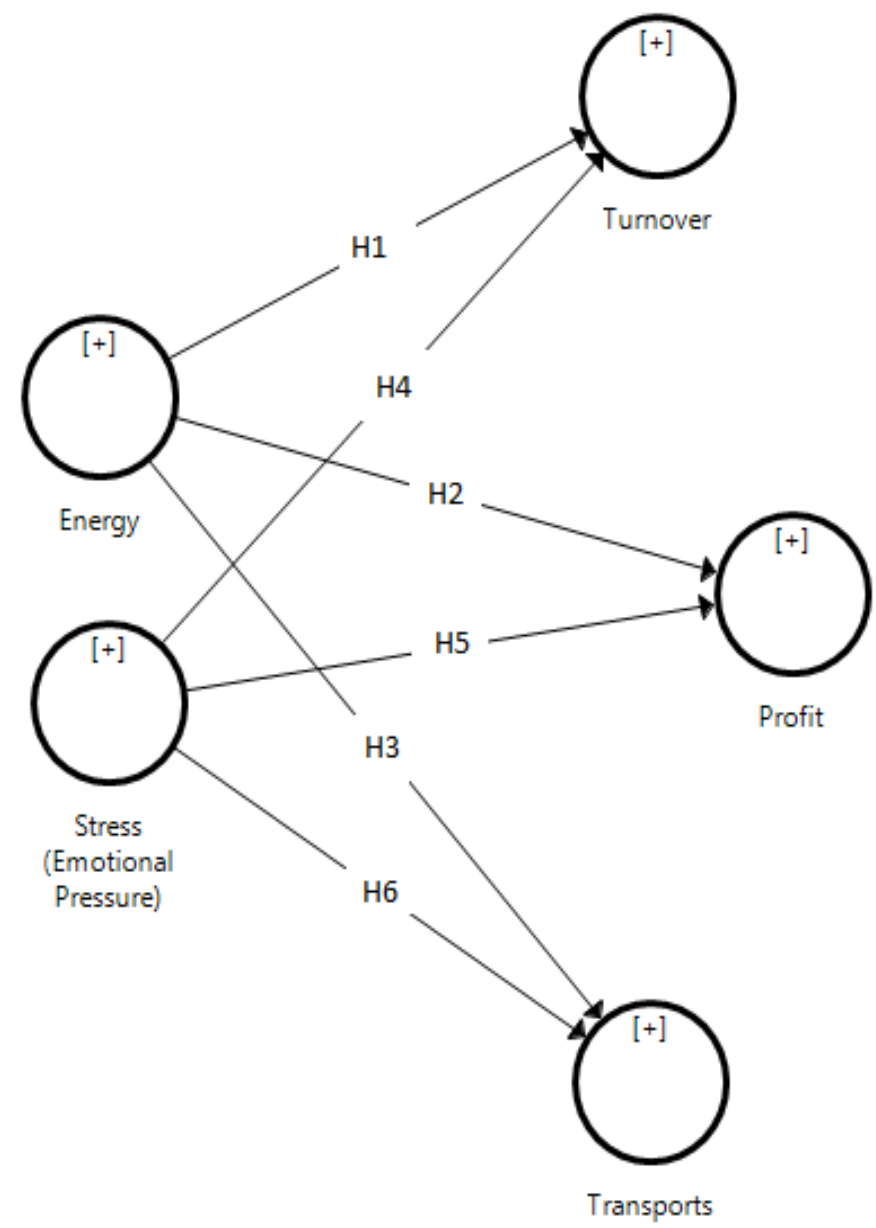

PICBE | 979

Figure 1. Conceptual model

Source: Authors' own research

And the model with the empirical data collected during a year and a half at a logistics company (Torp et al., 2017a) resulted in the following performance predictors, that is shown in figure 2 , for stress and energy respectively: 


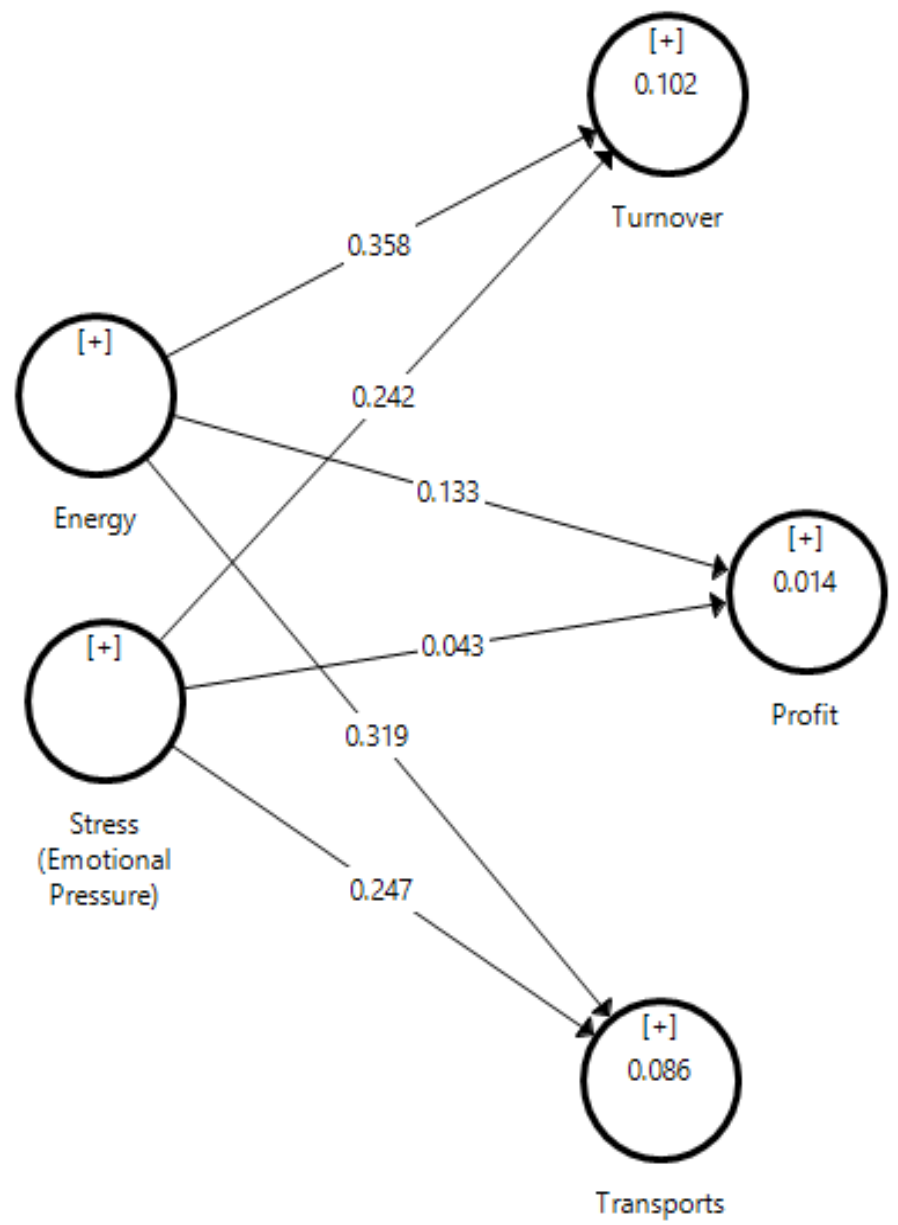

PICBE $\mid 980$

Figure 2. Stress and Energy as performance predictors

Source: Authors' own research.

As it can be seen, there is a clear connection between a person's energy level and at least certain of the included performance indicators. The same goes with the level of stress (emotional pressure). The level of energy that a person has predicts the future turnover generated by that person (Beta coeff. $=0.358$; $\mathrm{p}<0.05$ ), and the number of transports (Beta coeff. $=0.319 ; \mathrm{p}<0.05)$. Both are statistically significant, and, compared with the present array of HR performance indicators, relatively good in their prediction. Stress, or emotional pressure, was also found to be a predictor of future turnover (Beta coeff. $=0.242 ; \mathrm{p}<0.05$ ), and of number of transports generated (Beta coeff. $=0.247 ; \mathrm{p}<0.05$ ). Thus, stress is not as good a performance predictor as energy, however, both are statistically significant predictors. Neither stress nor energy have been found to offer any statistically valid prediction regarding profit, at least not directly.

Based on the presented data, it seems that the present array of HR performance predictors may be expanded with both energy and stress. Thus, the number of tools that contemporary companies possess to predict performance is enlarged. The interesting question, something that also Hunter \& Hunter (1984) touches, is the prediction that can be done based on two or more predictors. Hunter \& Hunter write that one should be careful when using more than one predictor, because the final prediction can be of lower accuracy using more performance predictors, than using just one. That happens, as they write, if the 
performance predictors are not rated according to their predictive ability. In regard to the present discussion, it might be that the predictions made based on a person's energy profile are completely different than predictions made based on other performance predictors, and thus gives more of a complementary assessment, instead of a substitution for the other assessment. Would it, for example, be possible to imagine that combining cognitive ability and energy would give a much deeper-level assessment of the future performance of an employee? The reason for this is that it would show both the "processing power" (cognitive ability) and the "battery" (energy potential), and this would be better than by combining any other regular performance predictors (their predictions, when combined, would not be very different in essence).

\section{Conclusion and the future of HR: assessment, or development?}

Based on the presented data it also seems reasonable to include both stress and energy in the list of contemporary performance predictors. Although neither of them offers a perfect prediction of future performance - which none of the present performance indicators do either - both energy and stress seems to have a good prediction ability.

Obviously, further studies need to be conducted in order to establish the predictions of energy and stress amongst larger groups of test subjects, however, it seems, based on the present findings, that such studies may be worth conducting.

As previously stated, is it important for a company to be able to predict the future performance of an employee, however, it may probably also be of significant importance for a company to help their employees to develop. At least that is what the essence of different vanguard HR practices that companies worldwide are implementing is all about. Studies by Torp et al., $(2015,2016)$ show that the energy level of an employee can be increased by deliberate effort, and at the same time that the stress level can be diminished. Thus, since it is considered that stress and energy are indicators of future performance, logically it must follow that actions that lead to, for example, an increase in a person's level of energy, indirectly also must lead to an increase in that person's professional performance. Thus, Human Resource Management can become more than just about predicting employees with high potential, but about helping them develop and become better and better with each passing day.

\section{References}

Bodanis, D. (2000). E=mc². Haase \& Søns Forlag.

Boxall, P. \& Purcell, J. (2011). Strategy and Human Resource Management. Palgrave Macmillan.

Hawking, SW. (2010). The Grand Design. Bantam Press. London.

Hunt, VV. (1996). Infinite mind: The Science of Human Vibrations of Consciousness. Malibu Publishing Co.

Hunter, J.E., \& Hunter, R.F. (1984). Validity and Utility of Alternative Predictors of Job Performance. Psychological Bulletin. Vol. 96. No. 1.

Korotkov, KG. (2002). Human Energy Field: study with GDV bioelectrography. Backbone Publishing Co., Fair Lawn, NJ, USA.

Korotkov, KG. (Ed.) (2004). Measuring Energy Fields: Current Research. Backbone Publishing Co. Fair Lawn, USA.

Lengnick-Hall ML., Lengnick-Hall, CA., Andrade, LS., \& Drake, B. (2009). Strategic human 
resource management: The evolution of the field. Human Resource Management Review 19.

Motoyama, H. (1978). Science and the Evolution of Consciousness. Autumn Press.

Schmitt, N. (2012). The Oxford Handbook of Personnel Assessment and Selection. Oxford library of psychology. Oxford University Press. Oxford.

Tiller, W. (199)7. Science and Human Transformation. Pavior. Torp, AM., Marosy, ZI, \& Purcarea, AA. (2015). Mindfulness - may diminish stress and increase energy. Network Intelligence Studies. NIS 5, Volume III, Issue 1.

Torp, A., Bunea, A., \& Cipu, C. (2016). Company Aikido - It Seems to be a Practical Method to Reduce Stress and Increase a Person's Energy. SEA - Practical Application of Science, Vol. IV, Issue 1 (10)

Torp, A., Andrei, A., Purcarea, AA. (2017a). The Relation Between an Employee's Energy Profile and that Person's Professional Performance. Strategica. Bucharest: Tritonic.

Torp, A. (2017). Predicting Company Financial Performance Based on the Energy Profile of the Employees by the Use of the Electrophotonic Imaging Device. SAMRO. The $11^{\text {th }}$ International Management Conference. 